SOI: $\underline{1.1 / \text { TAS }}$ DOI: $10.15863 /$ TAS International Scientific Journal Theoretical \& Applied Science

p-ISSN: 2308-4944 (print) $\quad$ e-ISSN: 2409-0085 (online)

Year: 2017 Issue: 09 Volume: 53

Published: $30.09 .2017 \quad \underline{\text { http://T-Science.org }}$
Sergey Alexandrovich Mishchik

Associate Professor, Candidate of Pedagogical Science,

Academician of International Academy TAS, Assistant professor Department of Physics,

State Maritime University Admiral Ushakov, Russia, sergei_mishik@mail.ru

SECTION 21. Pedagogy. Psychology. Innovation in Education

\title{
SYSTEMIC PROBLEMS ELECTRICAL CONDUCTIVITY OF THE LIQUID OF APPLIED PHYSICS MARITIME FLOT OF PEDAGOGOMETRIC ANALYSIS
}

\begin{abstract}
The basic principles of the system of problems electrical conductivity of the liquid in applied physics Navy pedagogometric analysis of the formation of mathematical models of learning activities about the nature of achieving the criteria of life, cycling, systemsness and phasing, which form a basic cell of the educational space, as well as prima nenie twelve pointed star Ertsgammy relatively presentation ertsgamming principle which determines the foundations pedagogometric through forming substantive methods of hyper-space professional life, psychological and educational activity theory, psycho-pedagogical system analysis and the theory of the formation of mental actions.

Key words: pedagogometric, vital activity, cyclicity, system, phase, star Erzgammy, electrical conductivity of the liquid, applied physics, marine fleet.

Language: Russian

Citation: Mishchik SA (2017) SYSTEMIC PROBLEMS ELECTRICAL CONDUCTIVITY OF THE LIQUID OF APPLIED PHYSICS MARITIME FLOT OF PEDAGOGOMETRIC ANALYSIS. ISJ Theoretical \& Applied Science, 09 (53): 164-170.

Soi: http://s-o-i.org/1.1/TAS-09-53-25 Doi: crossef https://dx.doi.org/10.15863/TAS.2017.09.53.25
\end{abstract}

УДК 372.851

\section{СИСТЕМНЫЕ ЗАДАЧИ ЭЛЕКТРОПРОВОДНОСТИ ЖИДКОСТИ ПРИКЛАДНОЙ ФИЗИКИ МОРСКОГО ФЛОТА ПЕДАГОГОМЕТРИЧЕСКОГО АНАЛИЗА}

Аннотация: Рассмотрены основные принщипь построения системных задач электропроводности жидкости прикладной физики морского флота педагогометрического анализа при формировании математических моделей учебной деятельности относительно характера достижения критериев жизнедеятельности, ичиклиности, системности и этапности, которые образуют базисную ячейку образовательного пространства, а также применение двенадиати конечной звезды Эригаммы относительно представления принципа эригаммности, который определит основы педагогометрики через формообразование предметными методами гиперпространства профессиональной жсизедеятельности, психолого-педагогической теории деятельности, психолого-педагогического системного анализа и теории формирования умственных действий.

Ключевые слова: педагогометрика, жизнедеятельность, ичикличность, системность, этапность, звезда Эригаммы, электропроводность жидкости, прикладная физика, морской флот.

\section{Introduction}

Формирование системных задач электропроводности жидкости прикладной физики морского флота педагогометрического анализа связывается с решением общей задачи педагогометрики математических моделей учебной деятельности на основе базисных представлений методологии педагогометрического анализа, отражающего особенности структуры и формы жизнедеятельности, цикличности, системности и этапности. В результате педагогометрического анализа создаётся базисная ячейка образовательного пространства, которая выражает принцип эрцгаммности через всеобщую структуру двенадцати конечной 
звезды Эрцгаммы. Представленная зависимость выражает основы педагогометрики через обособление предметных методов гиперпространства профессиональной жизнедеятельности, психолого-педагогической теории деятельности, психолого-педагогического системного анализа и теории формирования умственных действий $[1,2,3]$.

Выделенные основы подготовки инновационных широкопрофильных специалистов направлены на совершенствование базы предметных прикладных профессиональных задач электропроводности жидкости прикладной физики морского флота педагогометрического анализа, через целостную профессиональную деятельность на морском флоте. Представление математических моделей учебнопрофессиональной деятельности специалистов инновационного мышления ориентируется на: базисную звезду Эрцгаммы гиперпространства жизнедеятельности (Е1); базисный целостносистемный цикл жизнедеятельности (Е2); базисную звезду Эрцгаммы системного анализа (Е3); базисное проявление двенадцати этапов и форм познавательного гиперпространства жизнедеятельности образовательного процесса (E4) [4,5,6].

Формирование системных задач электропроводности жидкости прикладной физики морского флота педагогометрического анализа и адаптивной базы предметных педагогометрических моделей эрцгаммного анализа образовательных объектов с признаком базисно-нормативной эрцгаммности, представлют их обобщённые структуры. В результате реализуется собственная функция психолого-математического представления профессионально-значимых объектов системных задач электропроводности жидкости прикладной физики морского флота педагогометрического анализа через объелинение признаков смыслообразования учебно-профессионального действия, его принятия, ориентировочноисполнительно-контрольных признаков и прогноза совершенствования анализа объектов педагогометрческого содержания [7,8,9].

\section{Materials and Methods}

Системные задачи электропроводности жидкости прикладной физики морского флота отражают целостно-системное моделирование основных элементов транспортных объектов. При этом возникает ориентация на единство базисных характеристик предметных и исполнительных условий относительно предмета содержания и способа его реализации через представление базисной ячейки образовательного пространства, которая отражает принцип эрцгаммности адекватного структуре двенадцати конечной звезды Эрцгаммы. Рассматриваются: определение сопротивления морской воды, скорость движения ионов в электролите судового аккумулятора, условия эффективной зарядки и эксплуатации судовых аккумуляторов, причины возрастания плотности электролита судового кислотного аккумулятора, определение удельной электропроводности абсолютно чистой воды на морском флоте [10, 11,12].

В процессе решения системных задач электропроводности жидкости прикладной физики морского флота необходимо применять основные положения теории деятельности, системного анализа и теории формирования интеллекта через построение математических моделей учебно-профессиональной активности отражающей структуру: базисной звездой Эрцгаммы гиперпространства жизнедеятельности (E1); базисного целостно-системного циклом жизнедеятельности (Е2); базисной звездой Эрцгаммы системного анализа (Е3); базисного проявления двенадцати этапов и форм познавательного жизнедеятельности гиперпространства образовательного процесса (Е4).

Системный анализ предполагает выполнение последовательности системных аналитических действий: выделить объект анализа -задачу электропроводности жидкости прикладной физики морского флота (ЗЭЖПФМФ) как систему; установить порождающую среду ЗЭЖПФМФ; определить уровни анализа ЗЭЖПФМФ; представить целостные свойства ЗЭЖПФМФ относительно пространственных, и временных характеристик и их комбинаций; выделить структуру уровня анализа ЗЭЖПФМФ; установить структурные элементы уровня анализа ЗЭЖПФМФ; определить системообразующие связи данного уровня анализа ЗЭЖПФМФ; представить межуровневые связи анализа ЗЭЖПФМФ; выделить форму организации ЗЭЖПФМФ; установить системные свойства и поведение ЗЭЖПФМФ.

\section{Задача 1}

Два электрода-зонда судовой системы автоматического контроля в виде металлических шаров диаметром $\boldsymbol{d}=\mathbf{3 0} \boldsymbol{c \boldsymbol { m }}$ находятся в море на изолированных кабелях на глубине $\boldsymbol{h}=\boldsymbol{6 0} \boldsymbol{m}$. Расстояние между электродами-зондами судовой системы автоматического контроля $\boldsymbol{\ell}=300 \mathrm{~m}$. Удельная проводимость морской воды $\lambda=4$ $\boldsymbol{C M} / \boldsymbol{M}$. Определите сопротивление морской воды между электродами-зондами судовой системы автоматического контроля.

Ответ: $R=0,14 O$ M.

\section{Задача 2}


Определить скорость движения ионов в электролите судового аккумулятора, если концентрация ионов в электролите судового аккумулятора равна $\boldsymbol{n}=\mathbf{1 0}^{22} \boldsymbol{c m}^{-3}$, площадь каждого электрода судового аккумулятора $\boldsymbol{S = 5 0}$ $\boldsymbol{c m}^{2}$ и сила тока в судовой электрической цепи равна $I=1$ Ампер.

Ответ: $v=0,00013 \mathrm{~mm} / \mathrm{c}$.

\section{Задача 3}

Определить силу тока, проходящего через раствор электролита судового газоанализатора, если за $\boldsymbol{t}=1$ минуту разлагается $\boldsymbol{m}=1$ грамм воды. Определить объём гремучего газа, который выделяется в судовом газоанализаторе при нормальных условиях.

$$
\text { Ответ: } I=\frac{2 m F n}{M t}=178,7 \mathrm{~A} \text {. }
$$

\section{Задача 4}

Определить количество электроэнергии необходимой для получения в судовом газоанализаторе водорода объёмом $\boldsymbol{V}=2,5$ литра при температуре $\boldsymbol{t}=\mathbf{2 5}^{\circ} \boldsymbol{C}$ и давлении $\boldsymbol{P}=$ $10^{5}$ Паскалей, если электролиз ведётся при напряжении $\boldsymbol{U}=\boldsymbol{5}$ Вольт, а коэффициент полезного действия судового газоанализатора равен $\boldsymbol{\eta}=75 \%$.

$$
\text { Ответ: } W=\frac{2 \eta U F p V}{R T}=73 \kappa Д ж
$$

\section{Задача 5}

Определить Э.Д.С. $\boldsymbol{\varepsilon}$ элемента судового аккумулятора, если при соединении одного грамм-эквивалента цинка с серной кислотой выделяется $Q_{1}=124$ ккал тепла, а для выделения одного грамм-эквивалента меди из $\mathrm{CuSO}_{4}$ необходимо затратить $\boldsymbol{Q}_{2}=\mathbf{9 9 , 7}$ ккал энергии.

Ответ: Э.Д.С. $\varepsilon=1,06$ В.

$\begin{array}{ccc}\text { Падача } 6 & \\ \text { При } & \text { зарядке } & \text { судового }\end{array}$ аккумулятора плотность электролита увеличивается от $\rho_{1}=1,1 \mathrm{z} / \mathrm{cm}^{3}$ до $\rho_{2}=1,3 \mathrm{z} / \mathrm{cm}^{3}$. Объяснить причину возрастания плотности электролита судового кислотного аккумулятора.

Ответ: причины возрастания плотности представляются уравнением:

$$
\begin{aligned}
& \text { рарядка } \\
& \mathrm{PbO}_{2}+\mathrm{Pb}+2 \mathrm{H}_{2} \mathrm{SO}_{4} \frac{\longmapsto}{\text { зарядка }} 2 \mathrm{PbSO}_{4}+2 \mathrm{H}_{2} \mathrm{O} .
\end{aligned}
$$

\section{Задача 7}

Две вертикально расположенные параллельные пластины плоского датчикаконденсатора судовой системы автоматического управления касаются поверхности контрольной жидкости. Размеры пластин $\boldsymbol{a}$ и $\boldsymbol{b}$, расстояние между ними $\boldsymbol{d}$. Пластины плоского датчикаконденсатора судовой системы автоматического управления заряжены до разности потенциалов фо и отключены от источника судового электропитания. Определить высоту подъёма контрольной жидкости.

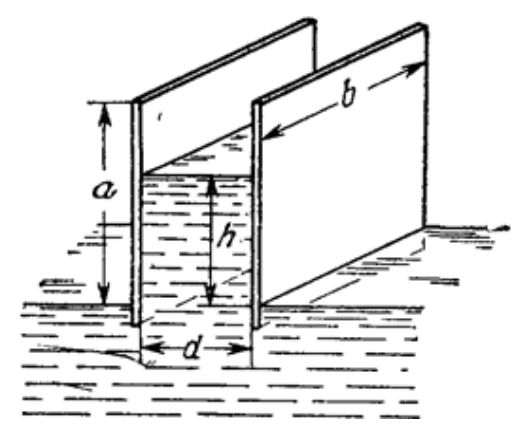

Рисунок 1 - Датчик-конденсатор судовой системы автоматического управления.

Ответ:

$$
h^{2}+\frac{a}{\varepsilon-1} h-\frac{\varepsilon_{o} a \varphi_{0}^{2}}{\rho g d^{2}}=0
$$

\section{Задача 8}

Две вертикально расположенные параллельные пластины плоского датчикаконденсатора судовой системы автоматического управления касаются поверхности контрольной жидкости. Размеры пластин $\boldsymbol{a}$ и $\boldsymbol{b}$, расстояние между ними $\boldsymbol{d}$. Пластины плоского датчикаконденсатора судовой системы автоматического управления заряжены до разности потенциалов фо и постоянно подключены к источнику судового электропитания. Определить высоту подъёма контрольной жидкости.

Ответ:

$$
\Delta W_{\text {пот }}=\frac{1}{2} \boldsymbol{m g h}=\frac{1}{2} \rho g b d h^{2} .
$$




\begin{tabular}{|c|c|c|c|c|c|c|}
\hline Impact Factor: & $\begin{array}{l}\text { ISRA (India) } \\
\text { ISI (Dubai, UAF } \\
\text { GIF (Australia) } \\
\text { JIF }\end{array}$ & $\begin{array}{l}=1.344 \\
=0.829 \\
=0.564 \\
=1.500\end{array}$ & $\begin{array}{l}\text { SIS (USA) } \\
\text { PИНЦ (Russia) } \\
\text { ESJI (KZ) } \\
\text { SJIF (Morocco) }\end{array}$ & $\begin{array}{l}=\mathbf{0 . 9 1 2} \\
=\mathbf{0 . 2 0 7} \\
=\mathbf{3 . 8 6 0} \\
=\mathbf{2 . 0 3 1}\end{array}$ & $\begin{array}{l}\text { ICV (Poland) } \\
\text { PIF (India) } \\
\text { IBI (India) }\end{array}$ & $\begin{array}{l}=6.630 \\
=1.940 \\
=4.260\end{array}$ \\
\hline
\end{tabular}

Задача 9

Определить расход цинка в судовом электрическом аккумуляторе за $\boldsymbol{t}=\mathbf{2 0}$ минуm работы при токе нагрузки $\boldsymbol{I}=\mathbf{0 , 5}$ Aмnер. Валентность цинка равна $\boldsymbol{n = 2}$. Принять, что «выход» тока судового электрического аккумулятора равен $\boldsymbol{\eta}=\mathbf{1 0 0 \%}$.

Ответ: $m=0,203$ грамма.

\section{Задача 10}

Для контроля качества работы судовой опреснительной установки определить удельную электропроводность абсолютно чистой воды при

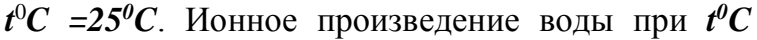
$=25^{\circ} \mathrm{C}$ равно $1,00 \cdot 10^{-14}$, mo есть $\mathrm{K} \cdot\left[\mathrm{H}_{2} \mathrm{O}\right]=\mathrm{Kв}=$

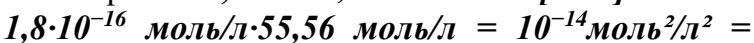
$[\mathrm{H}+] \cdot[\mathrm{OH}-]\left(\right.$ npu $\left.25^{\circ} \mathrm{C}\right)$.

Ответ: $\lambda=5.5 \times 10^{-6} \mathrm{CM}_{\mathrm{M}} \mathrm{M}^{-1}$

\section{Задача 11}

В судовой системе электронного контроля определить скорость движения иона $\mathrm{Na}+$ в водном растворе при $\boldsymbol{t}^{0} \boldsymbol{C}=\mathbf{2 5 ^ { 0 }} \boldsymbol{C}$, если разность потенциалов на электродах контрольного судового устройства равна $\Delta \varphi=10 \mathrm{~B}$. Электроды контрольного судового устройства находятся в водном растворе на расстоянии $\boldsymbol{d}=\boldsymbol{l} \boldsymbol{c} \boldsymbol{M}$ друг от друга. Определить время движения иона $\mathrm{Na}$ между электродами контрольного судового устройства

$$
\text { Ответ: } v=5,2 \cdot 10^{-5} \mathrm{M} / \mathrm{c} ; t=193 \mathrm{c} \text {. }
$$

\section{Задача 12}

Для судовой системы электронного контроля определить удельную электропроводность $\lambda$ морской воды (водного раствора $\mathrm{NaCl}$ ) объёмом $\boldsymbol{V}=\boldsymbol{1}$ литр при температуре $\boldsymbol{t}^{0} \boldsymbol{C}=\mathbf{2 5 ^ { 0 }} \boldsymbol{C}$ считая, что подвижности ионов при этой концентрации равны их предельным подвижностям. Определить расстояние, пройденное ионами $\mathrm{Na}+$ и $\mathrm{Cl}$ - за время $\boldsymbol{t}=10$ минуm в судовой системе электронного контроля, если через слой раствора длиной $\boldsymbol{\ell}=\boldsymbol{1} \boldsymbol{c m}$, заключенный между датчикамиэлектродами судовой системе электронного контроля площадью $S=1 \boldsymbol{c m}^{2}$, пропускают ток силой $\boldsymbol{I}=\mathbf{1} \boldsymbol{\mu} \boldsymbol{A}$.

Ответ: $\lambda=0,1264 \mathrm{Cm} \cdot \mathrm{M}^{-1} ; \ell(\mathrm{Na}+)=2,46$ м.м; $\ell(C l-)=3,75 \mathrm{Mm}$.

\section{Задача 13}

Для судовой системы электронного контроля определить эффективный радиус $\boldsymbol{r}$ иона $\mathbf{L i +}$ при температуре $\boldsymbol{t}^{0} \boldsymbol{C}=\mathbf{2 5}^{\boldsymbol{0}} \boldsymbol{C}$ из его предельной подвижности, используя закон Стокса. Определить приблизительное число $\boldsymbol{n}$ молекул воды, входящих в гидратную оболочку иона $\mathbf{L i +}$. Кристаллографический радиус иона $\boldsymbol{L} \boldsymbol{i}+$ равен $\boldsymbol{r}=$ $60 \mathrm{~nm}$. Вязкость воды при температуре $\boldsymbol{t}^{0} \mathrm{C}=\mathbf{2 5}^{\mathbf{0}} \mathrm{C}$ равна $\boldsymbol{\eta}=\boldsymbol{8 , 9 1} \cdot 10^{-4} \boldsymbol{\Pi a} \boldsymbol{c}$. Собственный объем молекулы воды оценить из параметров уравнения Ван-дер-Ваальса.

$$
\text { Ответ: } r=2,5 A^{0} ; \quad n\left(\mathrm{H}_{2} \mathrm{O}\right)=5 \text {. }
$$

\section{Задача 14}

Удельная электропроводность водного раствора сильного электролита судовой системы электронного контроля при температуре $\boldsymbol{t}^{0} \boldsymbol{C}=\mathbf{2 5 ^ { 0 }} \boldsymbol{C}$ равна $\lambda_{1}=109,9 \boldsymbol{C M}_{\boldsymbol{M}} \cdot \boldsymbol{c m}^{2} \cdot \boldsymbol{M o л ь}^{-1}$ при концентрации $n_{1}=6,2 \cdot 10^{-3}$ моль ${ }^{-1}$ и $\lambda_{2}=106,1 \mathrm{Cм}^{\circ} \mathrm{cm}^{2} \cdot$ моль $^{-1}$ при концентрации $\boldsymbol{n}_{2}=1,5 \cdot 10^{-2} \quad$ моль $\cdot \pi^{-1}$. Определить удельную электропроводность раствора судовой системы электронного контроля при бесконечном разбавлении раствора электролита.

$$
\text { Ответ: } \lambda=116,7 \mathrm{Cm}^{\prime} \mathrm{cm}^{2} \cdot \text { моль }^{-1} \text {. }
$$

\section{Задача 15}

Для судового гальванического элемента определить изменение энтальпии $\boldsymbol{\Delta H}$ и количество теплоты $\boldsymbol{q}$, выделяющейся в судовом гальваническом элементе при обратимом протекании в водном растворе реакции $\mathrm{CuSO}_{4}+$ $\boldsymbol{Z n}=\boldsymbol{C u}+\mathbf{Z n S O}_{4}$, если при температуре $\boldsymbol{T}_{\boldsymbol{l}}=\mathbf{2 7 3}$ К, э.д.c. $\varepsilon_{1}=1,0960$ В, а при $T_{2}=276$ К, э.д.c. $\varepsilon_{2}=$ $1,0961 \mathrm{~B}$.

Ответ: $\Delta H=-2,1 \cdot 10^{8}$ Дюж; $q=1,755 \cdot 10^{6}$ Джс/кг-экв.

\section{Задача 16}

Плотность раствора серной кислоты в заряженном судовом элементе свинцового аккумулятора $\boldsymbol{d}_{3}=1,28 \mathrm{z} / \mathrm{cm}^{3}$ (концентрация $36,87 \%$ по массе), в разряженном судовом элементе свинцового аккумулятора $\boldsymbol{d}_{p}=1,10 \mathrm{z} / \mathrm{cm}^{3}$ (концентрация $\mathbf{1 4 , 3 5 \%}$ по массе). Определить количество электролита необходимое для заливки полностью разряженного элемента судового свинцового аккумулятора с фактической ёмкостью $\boldsymbol{Q \phi a \kappa m}=120 \boldsymbol{A} \cdot \boldsymbol{\varphi} \boldsymbol{a c}$, чтобы изменение плотности его электролита в процессе зарядаразряда находилось в указанных пределах. Определить коэффициент использования $\mathrm{H}_{2} \mathrm{SO}_{4}$.

Ответ: $V=1240$ мл; Кисn $=69,2 \%$.

Определить объём электролита для последовательно соединённой судовой аккумуляторной батареи номинальной ёмкостью $Q=1,5 A \cdot 4 a c$, состоящей из $n=102$ элементов, если «полезный» коэффициент использования кислоты в судовой аккумуляторной батареи равен Kucn $=55 \%$. Начальная концентрация $\mathrm{H}_{2} \mathrm{SO}_{4} \mathrm{C}=392$ г/л, электрохимический эквивалент $\mathrm{H}_{2} \mathrm{SO}_{4} q=2,20$ 2/ $\mathrm{A} \cdot 4 \mathrm{ac}$.

Ответ: $V=1560$ мл. 


\section{Conclusion}

Условия формирования и развития инновационного широкопрофильного профессионального мышления базируется на организации всестороннего развития педагогометрической эрцгаммности. Представленные системные задачи электропроводности жидкости прикладной физики морского флота педагогометрического анализа выделяют основные направления развития и совершенствования базы прикладных предметных педагогометрических моделей образовательных объектов относительно педагогометрического математического моделирования учебного процесса. Формируемая образовательная деятельность связывается с процессами совершенствования программируемых математических моделей учебной активности относительно характера достижения критериев жизнедеятельности, цикличности, системности и этапности.

Разнообразные содержательные задачи педагогометрического анализа ориентируются на специальные статистические выборки данных учебного процесса, характеризующих исследуемые образовательные явления и представленных во времени в форме временных рядов.

Для моделирования тенденции развития педагогометрических процессов и явлений можно применять кривые дидактического роста, которые позволяют определить педагогометрическую функцию поведения показателя в предыдущих периодах анализа, чтобы выявить возможное поведение педагогометрического показателя в будущем, который зависит лишь от временной переменной $[13,14,15]$.

Данная функция времени - кривая дидактического роста позволяет получить выравненные, теоретические значения уровней временного ряда. которые наблюдались, если бы тенденция педагогометрического процесса полностью совпадала с кривой. Эта позволяет применить функции дидактического роста для прогнозирования педагогометрических процессов.

Педагогометрическое прогнозирование на основе кривых дидактического роста основано на экстраполяции. При этом предполагается, что во временном ряде присутствует тренд и характер развития показателя обладает свойствами инерционности.

Процесс разработки педагогметрического прогноза с использованием кривых дидактического роста сводится к этапам: выбор кривых роста, форма которых соответствует характеру изменения временного педагогометрического ряда; оценка параметров выбранных кривых по параметрам соответствия базисной звезде Эрцгаммы гиперпространства жизнедеятельности (Е1); базисному целостно-системным циклу жизнедеятельности (E2); базисной звезде Эрцгаммы системного анализа (Е3); базисному проявлению двенадцати этапам и форм познавательного гиперпространства жизнедеятельности относительно образовательного процесса (E4); проверка адекватности выбранных кривых прогнозируемому образовательному процессу.

Существуют различные типы кривых дидактического роста, применяемых для моделирования педагогометрических процессов. Эти кривые могут быть разделены на три класса в зависимости от того, какой тип динамики развития образовательного объекта они представляют.

К первому классу кривых дидактического роста относятся функции, используемые для представления процессов без предела роста. К этому классу функций относятся полиномиальные кривые и простая экспоненциальная кривая.

Ко второму классу кривых дидактического роста относятся функции, применяемые для представления процессов с пределом роста без точки перегиба. К этому классу педагогометрических функций относятся модифицированная экспонента.

К третьему классу кривых дидактического роста относятся функции, используемые для представления процессов с пределом роста и имеющие точку перегиба. К этому классу педагогометрических функций относятся кривая Гомперца и логистическая кривая.

Основные характеристики отдельных типов дидактических кривых в педагогометрическом моделировании отражают кинематические параметры образовательных процессов.

Общий вид полиномов дидактических процессов следующий:

$$
y_{t}=a_{0}+a_{1} t+a_{2} t^{2}+\ldots+a_{q} t^{q},
$$

где $a_{0}, a_{1}, a_{2}, \ldots a_{q}$ - параметры полинома.

Параметры дидактических полиномов имеет конкретную интерпретацию в зависимости от содержания временного ряда педагогометрического анализа. Определение: скорости дидактического роста - параметр $a_{1}$, ускорения дидактического роста - параметр $a_{2}$, изменения ускорения дидактического роста параметр $a_{3}$, начальное состояние дидактического процесса - параметр $a_{0}$.

Данная классификация позволяет организовать многомерное построение базисной 
звезды Эрцгаммы гиперпространства жизнедеятельности (Е1); базисного целостносистемным цикла жизнедеятельности (Е2); базисной звездой Эрцгаммы системного анализа (Е3); базисного проявления двенадцати этапов и форм познавательного гиперпространства жизнедеятельности относительно образовательного процесса (E4) и увязать с моделями временных педагогометрических рядов, которые требуют корректировки сезонных эффектов и сглаживания.

Установленные процессы педагометрического анализа образуют базисную ячейку образовательного пространства и отражают смысл двенадцати конечной звезды Эрцгаммы относительно представления принципа эрцгаммности. Выделенные структуры определяют основы педагогометрики через установление предметными методами гиперпространства профессиональной жизнедеятельности, психолого-педагогической теории деятельности, психолого-педагогического системного анализа и теории формирования умственных действий. Представленные критерии жизнедеятельности, цикличности, системности и этапности, которые формируют базисную ячейку образовательного пространства, создают условия развития абсолютного инновационного образовательного цикла, отражающего специфическую структуру подготовки широкопрофильно-инновационных специалистов при реализации международных образовательных стандартов эревнометрического содержания алигорамной формы.

\section{References:}

1. Mishchik SA (2014) Pedagogometrika and mathematical modeling educational activity. Materialy Mezhdunarodnoy nauchnoy konferenctsii "Modern mathematics in science" - 30.06.2014. ISJ Theoretical \&Applied Science 6(14): 54-56 Caracas, Venezuela. doi: http://dx.doi.org/10.15863/TAS.2014.06.14.10

2. Mishchik SA (2014) Simulation training activity methods of mathematical logic. Materialy Mezhdunarodnoy nauchnoy konferenctsii "Eurapean Science and Education" - 30.07.2014. ISJ Theoretical \&Applied Science 6(15): 72-74 Marseille, France. doi: http://dx.doi.org/10.15863/TAS.2014.07.15.13

3. Mishchik SA (2014) Mathematical modeling system integrity-cycle of life activity - first goal pedagogometriki. Materialy Mezhdunarodnoy nauchnoy konferenctsii "European Applied Sciences" - 30.08.2014. ISJ Theoretical \&Applied Science 7(16): 77-79. Aix-en-Provence, France. doi: http://dx.doi.org/10.15863/TAS.2014.08.16.13

4. Mishchik SA (2014) Mathematical modeling system integrity-curricular activities - the second problem pedagogometriki. Materialy Mezhdunarodnoy nauchnoy konferenctsii "European Innovation" - 30.09.2014. ISJ Theoretical \&Applied Science 9(17): 126-128 Martigues, France. doi: http://dx.doi.org/10.15863/TAS.2014.09.17.21

5. Mishchik SA (2014) Mathematical modeling holistic-systemic communicative activity - the third task pedagogometriki. Materialy
Mezhdunarodnoy nauchnoy konferenctsii "European Scientific Achievements" 30.10.2014. ISJ Theoretical \&Applied Science 10(18): 45-47 Brighton, UK. doi: http://dx.doi.org/10.15863/TAS.2014.10.18.11

6. Mishchik SA (2014) Mathematical modeling integrity - system performance subject - fourth task pedagogometriki. Materialy Mezhdunarodnoy nauchnoy konferenctsii "Eurapean Science and Technology" 30.11.2014. ISJ Theoretical \&Applied Science 11(19): 51-54 Southampton, UK. doi: http://dx.doi.org/10.15863/TAS.2014.11.19.10

7. Mishchik SA (2015) Pedagogometrik - science and academic subject. Materialy Mezhdunarodnoy nauchnoy konferenctsii "European Technology in Science" 28.02.2015. ISJ Theoretical \& Applied Science 02 (22): 103-106 Malmö, Sweden. doi: http://dx.doi.org/10.15863/TAS.2015.02.22.17

8. Flyorov V.N. Sbornik zadach po prikladnoj elektroximii. M.: Vysshaya shkola, 1967 S.292.

9. Tokmazov GV (2014) Matematicheskoe modelirovanie $\mathrm{v}$ uchebno-professional'noy deyatel'nosti. Materialy Mezhdunarodnoy nauchnoy konferentsii «Modern mathematics in science» - 30.06.2014. ISJ Theoretical \& Applied Science 6(14): 44-46. - Caracas, Venezuela. doi: http://dx.doi.org/10.15863/TAS.2014.06.14.8

10. Tokmazov GV (2014) Mathematical modeling research skills in educational activity methods of probability theory. Materialy 


\begin{tabular}{l|lrl|l|ll} 
& ISRA (India) & $=\mathbf{1 . 3 4 4}$ & SIS (USA) & $=\mathbf{0 . 9 1 2}$ & ICV (Poland) & $=\mathbf{6 . 6 3 0}$ \\
Impact Factor: & ISI (Dubai, UAE) $=\mathbf{0 . 8 2 9}$ & PUHL (Russia) $=\mathbf{0 . 2 0 7}$ & PIF (India) & $=\mathbf{1 . 9 4 0}$ \\
& GIF (Australia) & $\mathbf{0 . 5 6 4}$ & ESJI (KZ) & $=3.860$ & IBI (India) & $=\mathbf{4 . 2 6 0}$ \\
& JIF & $\mathbf{1 . 5 0 0}$ & SJIF (Morocco) & $=\mathbf{2 . 0 3 1}$ & & \\
\hline
\end{tabular}

Mezhdunarodnoy nauchnoy konferenctsii "Eurapean Science and Technology" 30.11.2014. ISJ Theoretical \&Applied Science 11(20): 66-69 Southampton, United Kingdom. doi:

http://dx.doi.org/10.15863/TAS.2014.11.19.13

11. Mishhik N.A. Pravovy'e osnovy' francuzskoj sistemy' bor'by' s zagryazneniem morya / Nauchny'e issledovaniya: Informaciya, analiz, prognoz [Tekst]: monografiya / [V.E'.Lebedev, A.A.Sviridenko, V.M.Sokolinskij i dr.]; pod obshhej red. prof. O.I.Kirikova - Kniga 51.Voronezh-Moskva, 2016.

12. Mishchik NA (2014) The practice of french justice article 228 of the UN convention on the law of the sea. Materialy Mezhdunarodnoy nauchnoy konferenctsii "The European Science and Education"- 30.07.2014. ISJ Theoretical \&
Applied Science 07 (15): 93-97. - Marseille, France.

doi:

http://dx.doi.org/10.15863/TAS.2014.07.15.19

13. Mishhik N.A., Antonenko G.A. Liniya gorizonta kak gradientny'j perepad V fotograficheskix izmereniyax dlya celej morexodnoj astronomii//E'kspluataciya morskogo transporta. 2013. № 2 (72). Novorossijsk, p. 23-28.

14. 14.Mishhik, N.A. Optimizaciya metodov morexodnoj astronomii [Tekst]: avto-ref.dis. ... kand. tex. nauk: 05.22.16 / N.A.Mishhik. Novorossijsk, 2000. - $24 \mathrm{p}$.

15. 15.Mishhik, N.A. Optimizaciya metodov morexodnoj astronomii [Tekst]: dis. ... kand. tex. nauk: 05.22.16 / N.A.Mishhik. Novorossijsk, 2000. - 188 p. 